\title{
Preliminary Examination of Olanzapine and Diet Interactions on Metabolism in a Female Macaque
}

\author{
Oleg Varlamov ${ }^{1,2}$, Paul Kievit ${ }^{2}$, Kenny Phu ${ }^{1}$, Arubala P. Reddy ${ }^{1}$, Charles T. Roberts Jr ${ }^{1,2}$ and Cynthia L. \\ Bethea $^{1,3 *}$ \\ ${ }^{1}$ Divisions of Reproductive and Developmental Sciences, Oregon National Primate Research Center, Beaverton, OR 97006, USA \\ ${ }^{2}$ Division of Diabetes, Obesity, and Metabolism, Oregon National Primate Research Center, Beaverton, OR 97006, USA \\ ${ }^{3}$ Department of Obstetrics and Gynecology, Oregon Health and Science University, Portland, OR 97201, USA
}

Received: August 02, 2014; Accepted: September 12, 2014; Published: September 29, 2014

*Corresponding author: Cynthia L. Bethea, Division of Reproductive and Developmental Sciences, Oregon National Primate Research Center 505 NW 185th Ave., Beaverton, Tel: 503-690-5327; E-mail: betheac@ohsu.edu

\begin{abstract}
Clinical data suggest that atypical antipsychotics such as Olanzapine (OLZ) induce significant metabolic changes that are serious side effects of their primary use. Since controlled human studies are problematic and rodent data may be poorly translatable, we have initiated development of a macaque model of OLZ-induced metabolic disease. In this preliminary feasibility study, we examined some metabolic effects of OLZ in a female macaque in the context of a standard low-calorie/fat monkey chow diet followed by a highfat/sugar western-style diet (WSD). A female Japanese macaque was administered OLZ (1.25 mg/day) for 6 months, with dietary changes at 2-month intervals as follows: OLZ + Restricted chow, OLZ + Unrestricted chow, OLZ + WSD, and Placebo + WSD. Weight was assessed weekly. Glucose tolerance tests (GTT) and dexascans were performed at baseline and every 2 months. Omental (OM) and subcutaneous (SQ) adipose tissue biopsies were obtained at baseline, after OLZ + Unrestricted chow and after OLZ + WSD to evaluate adipocyte size, lipolysis, and insulin-stimulated free fatty acid uptake (FFA). A separate trial was conducted on two monkeys with 5 days of OLZ or no-treatment followed by RT-PCR on rostral and medial basal hypothalamus. Weight increased on administering OLZ + Restricted chow and stabilized on administering OLZ + Unrestricted chow. OLZ + WSD diet did not significantly change the weight plateau. Weight declined upon withdrawal of OLZ with continued WSD. Body fat increased from $14 \%$ at baseline to $22 \%, 30 \%, 28 \%$ and $19 \%$ at 2, 4, 6 and 8 months (mo), respectively, indicating that body fat was elevated on OLZ administration regardless of diet and declined upon OLZ removal. Glucose tolerance and the insulin response during GTT were normal with OLZ + Restricted chow or OLZ + Unrestricted chow diets. Addition of WSD with OLZ impaired glucose clearance during GTT. Insulin remained in the normal range, but first-phase insulin secretion was reduced. After removal of OLZ, but continued WSD administration, glucose clearance returned to normal; however, this was associated with hyperinsulinemia. Adipocyte diameter was increased in OM and SQ fat by OLZ + chow and OLZ + WSD to a similar extent ( $p<0.01,2$-way ANOVA). In OM, isoproterenol-stimulated lipolysis occurred at baseline. In both depots, isoproterenol-stimulated lipolysis occurred with OLZ + chow, but it was significantly blunted by addition of WSD (ANOVA $p$ $<0.0001$; post hoc $p<0.05$ ). Insulin increased FFA uptake at baseline. OLZ + chow or OLZ + WSD increased basal FFA uptake but insulininduced FFA uptake was blunted in both depots (post hoc $p<0.05$ ).
\end{abstract}

There was a marked decrease in pre-opiomelanocortin (POMC) gene expression, and increased agouti-related peptide (AgRP) and neuropeptide Y (NPY) expression in the hypothalamus. There was also a clear increase in serotonin (5HT) 2C, melanocortin (MCR4), and leptin (LepR) receptor gene expression. These data support the hypotheses that OLZ acts on peripheral tissues as well as in the CNS; that changes in hypothalamic gene expression occur very rapidly and precede increased fat accumulation; that adipose tissue exhibits insulin resistance prior to alterations in GTT; that addition of WSD to OLZ precipitates hyperglycemia without an obvious insulin response; and that removal of OLZ and continued WSD resulted in normalized glucose clearance and elevated insulin. These data suggest complex and early responses to OLZ that may be exacerbated by WSD.

Keywords: Olanzapine; Macaque; Western style diet; Insulin; Diabetes; POMC; AgRP, Free fatty acid; Lipolysis

\section{Introduction}

The twin epidemics of obesity and diabetes threaten to overwhelm healthcare systems in the U.S. and across all other parts of the world [1]. Specifically, 2/3 of the US population is overweight or obese, while $40 \%$ exhibit symptoms of pre- or frank diabetes and the combined direct and indirect costs of obesity and diabetes are now approaching $\$ 500$ billion a year. Thus, a better understanding of factors that contribute to their incidence is critical in order to manage this major public health issue. Important contributors to obesity and diabetes are lack of exercise and consumption of a high-fat/calorie Western-Style Diet (WSD). Compounding those factors in a subset of mentally ill patients is the use of Second-Generation Antipsychotics (SGAs), which are known to induce weight gain and to exacerbate risk for metabolic disease and diabetes, especially in youth.

The patients most often prescribed SGAs, have schizophrenia. Schizophrenia is a serious, non-curable mental illness with high morbidity and premature mortality [2]. It is generally estimated that today only approximately $10 \%$ to $15 \%$ of people who have schizophrenia are able to maintain full-time employment of any type, even with medication. The total indirect excess costs in the US were estimated to be $\$ 32.4$ billion in 2005 [3]. Adding 
metabolic disease on top of mental illness may be devastating and it decreases compliance. Currently psychiatrists prescribe SGAs, while these patients typically see an endocrinologist separately to manage their metabolic disease.

Nonetheless, the development and widespread use of SGAs has significantly improved the treatment and management of schizophrenia without the extra-pyramidal side effects of first generation antipsychotics such as haloperidol [4]. The older antipsychotics mainly acted as antagonists of D2 dopamine receptors [5]. SGAs exhibit lesser action at dopamine receptors and act more as serotonin (5HT) 2A/C receptor antagonists [6-9]. One widely used SGA is olanzapine (OLZ), which remains one of the most efficacious psychiatric medications in spite of its nearly universal metabolic side effects [10]. The SGAs ziprasidone and aripiprazole have reduced metabolic side effects, but also poorer scores on tests of positive and negative symptom relief $[11,12]$. Often ignored, however, is the fact that in the US, OLZ is taken by patients who typically consume a high-calorie/fat WSD. Little is known of the interaction between WSD and SGAs, and this variable is nearly impossible to test in humans.

Although OLZ may act at a number of receptors, the paramount role of the 5HT2C receptor in the hypothalamic feeding and satiety neuronal systems is undisputed [13]. Thus, the antagonism of the hypothalamic 5HT2C receptor by OLZ, probably plays a pivotal role. Most studies of the peripheral effects of OLZ in the area of metabolism have been conducted with rodents, but OLZ treatment has markedly different effects in rats and humans. OLZ regulates insulin secretion in islets and increases serum glucose in rats and humans, but there were serious inconsistencies in effects on weight gain, lipid concentrations, and Leptin levels in drug-treated rats $[14,15]$. Male rats showed an increased preference for a high-fat or -sugar diet, but did not exhibit greater weight gain than diet-matched controls [14], although female rats appear to be more sensitive to OLZ-induced weight gain [15]. However, there are no sex differences in humans. Thus, the absence of reliable effects of SGAs in rats indicates the poor predictive value of the rodent models.

Nonhuman primates (NHPs), however, are an exceptional model for human neuropsychiatry and metabolism. Monkeys in captivity eat monkey chow, which is very low in fat and sugar and high in micronutrients. We hypothesize that OLZ acts through both central and peripheral mechanisms that involve antagonism of 5HT2C receptors. We further hypothesize that the metabolic reactions to OLZ differ depending on diet. We have collected preliminary data from one longitudinally treated macaque with multiple weightings; Glucose Tolerance Tests (GTTs), Dexascans and adipose biopsies. The monkey responded to OLZ in a manner similar to humans, and the metabolic responses to OLZ differed significantly between a chow diet and a WSD. It appears that macaques are an excellent model for revealing the mechanisms by which OLZ induces metabolic syndrome in patients.

\section{Materials and Methods}

This experiment was approved by the IACUC of the Oregon National Primate Research Center and conducted in accordance with the with the NIH guideline (Guide for the Care and Use of Laboratory Animals, Eighth edition, NRC 2011).

\section{Animal}

An adult female Japanese macaque (Macaca fuscata) was housed in a large double cage for the duration of this study. The animal was maintained at a healthy weight and metabolic status; with a restricted, but adequate, diet of normal monkey chow (hereafter called 'chow') supplemented with fresh fruits and vegetables. Water was available ad libitum. Research veterinarians monitored the monkey continually.

\section{Diets}

Chow (Lab Diet 5000) contains $4.94 \mathrm{kcal} / \mathrm{g}$ and calories are distributed as $27 \%$ from protein, $14 \%$ from fat, and $58 \%$ from carbohydrates. Of the total $2.87 \mathrm{kcal} / \mathrm{g}$ of carbohydrates, $26 \%$ are derived from starch and $2.8 \%$ from sucrose. WSD (Lab Diet, TAD 5L0P) contains $4.94 \mathrm{kcal} / \mathrm{g}$ and the calories are distributed as $18 \%$ protein, $36 \%$ fat, and $46 \%$ carbohydrates. Of the total 3.36 $\mathrm{kcal} / \mathrm{g}$ of carbohydrates, $19.5 \%$ is derived from starch, $8.81 \%$ from sucrose, $4.41 \%$ from glucose, and $4.61 \%$ from lactose.

\section{Protocol}

The animal was weighed weekly throughout the protocol. Prior to any medication or diet manipulations, baseline GTT, Dexascan, and adipose tissue biopsies were obtained. After completing and recovering from biopsy surgery (described below), OLZ was administered at $1.25 \mathrm{mg}$ /day by placing the dissolving form in a food treat based on a human dose of $5 \mathrm{mg} /$ day for a $70 \mathrm{~kg}$ human. After 2 mo OLZ + Restricted chow, another GTT and dexascan were obtained, and the diet was changed to unrestricted monkey chow. This was accomplished by always maintaining extra chow biscuits in the cage. After 2 mo OLZ + Unrestricted chow, another GTT, dexascan, and a second biopsy were obtained. Upon recovery from biopsy surgery, the food was changed to WSD. After 2 mo OLZ + WSD, another GTT, dexascan, and a final biopsy (third) were obtained. She was then withdrawn from OLZ, and maintained for an additional 2 mo on WSD alone. After 2 mo WSD alone, another GTT and dexascan were obtained. The animal was then released from the protocol and returned to normal monkey chow.

\section{Euthanasia}

After 6 months on normal monkey chow, the same animal was administered OLZ for 5 days and euthanized by an expert veterinary pathologist in accordance with the American Veterinary Association guidelines (AVMA Guidelines for the Euthanasia of Animals, 2013 edition). She was transported to the necropsy suite under sedation, given an overdose of pentobarbital (30 mg/kg, i.v. (Hospira, Lake Forest, IL)) and exsanguinated with severance of the descending aorta. The brain was harvested, blocked, frozen in liquid $\mathrm{N}_{2}$ and stored at $-80^{\circ} \mathrm{C}$. An additional hypothalamus was obtained from an untreated female Japanese macaque that was euthanized for clinical reasons.

\section{Biopsy}

All surgical procedures were conducted by trained Surgical 
Services Unit personnel under the supervision of surgical veterinarians in dedicated surgical facilities using aseptic techniques and comprehensive physiological monitoring. All procedures took place in the operating rooms of the Surgical Services Unit. The animal had 22-gauge cephalic catheters placed, and was intubated in dorsal recumbency with a 4.06.0 ET tube. The animal was administered intravenous fluids (Lactated Ringers solution, $10 \mathrm{ml} / \mathrm{kg} / \mathrm{hr}$ ). Continuous monitoring was performed during the surgery for body temperature via esophageal temperature probe, heart rate, and pulse character (fast or slow) via pulse oximetry and electrocardiography, blood pressure via indirect blood pressure cuff or direct percutaneous arterial line, respiratory rate and pattern, end tidal carbon dioxide, capillary refill time, absence of palpebral response to touching the medial canthus, jaw tone and color of mucous membranes at gums or conjunctiva. A Verres needle was inserted via a $1-\mathrm{cm}$ sub umbilical skin incision followed by insufflations to $15 \mathrm{~mm} \mathrm{Hg}$ pressure with $\mathrm{CO}_{2}$ gas. The Verres was removed and the 11-mm trocar/sheath and 10-mm telescope was inserted by puncture at the same site. A right paralumbar 5-mm accessory port was placed, through which a cutting biopsy grasper was inserted. Pinch biopsy forceps were used to retrieve two fat biopsies from the falciform ligament. Grasping forceps were used to grab a small section of omentum, which was pulled through the side port. A $1 \times 2 \times 1$-cm block of omentum was removed via sharp and blunt dissection. The laparoscopic instruments were removed. A subcutaneous fat biopsy was then retrieved from the site of the scope incision. The tissue was placed in sterile culture medium for transport to the laboratory. The incisions were closed with interrupted 4-0 monocryl in the rectus fascia and skin. Recovery was on the OR table until extubation. Additional heat and oxygen support was provided as needed during the recovery period.

\section{Adipose tissue explant protocol}

The protocol for transport and processing of adipose tissue explants has been previously described [16-18]. The explants were incubated with and without insulin, in the presence of Isoproterenol or free fatty acids (FFA). For isoproterenolstimulated lipolysis studies, $100 \pm 10 \mathrm{mg}$ tissue explants were placed into a 24-well culture dish containing $0.5 \mathrm{~mL}$ incubation medium (phenol red-free DMEM [Invitrogen], 0.5\% BSA [SigmaAldrich], $20 \mathrm{mM}$ HEPES buffer [pH 7.4]), and incubated at $37^{\circ} \mathrm{C}$ free-floating for 2 hours in an atmosphere of $5 \% \mathrm{CO}_{2}$ at $37^{\circ} \mathrm{C}$. Glycerol release was determined using a colorometric glycerol detection kit (Zen-Bio). For determination of FFA uptake, fluorescent FFAs (BODIPY-C12; Invitrogen) were added to the culture medium.

\section{Confocal microscopy}

Intracellular fluorescence associated with BODIPY-C12 (FFA uptake) was determined as previously described with minor modifications [16-18]. Image recording and cell size determination were conducted using a Leica SP5 AOBS spectral confocal system as described [17].

\section{Data analysis}

Statistical analyses were not performed on the data collected in vivo since a single subject was studied for this preliminary examination and feasibility study. Multiple adipose explants were used for each measurement enabling legitimate statistical comparisons. Differences between groups were determined with two-way ANOVA followed by Bonferroni post hoc pair wise comparisons using Prism version 5 (Graph Pad Software, Inc., San Diego, CA).

\section{Results}

A longitudinal experimental design was implemented as described above Figure 1; top illustrates the experimental design, the timing of OLZ and dietary treatments, adipose biopsies, GTTs, dexascans and body weight. There was a rapid increase in weight on OLZ + Restricted chow that plateaued after OLZ + Unrestricted chow. Addition of WSD did not further increase weight. Unfortunately, there was a problem with palatability in this batch of WSD that was discovered subsequent to the experiment. Therefore, this particular piece of the data must be interpreted with caution. Nonetheless, weight reached a plateau on OLZ + Unrestricted chow that may have been maintained, or even increased, with more palatable WSD.

The Area Under Curve (AUC) for glucose and insulin secretion from the GTT data are shown in Figure 1, middle and bottom panels, respectively. The raw GTT data are shown in Figure 2. The
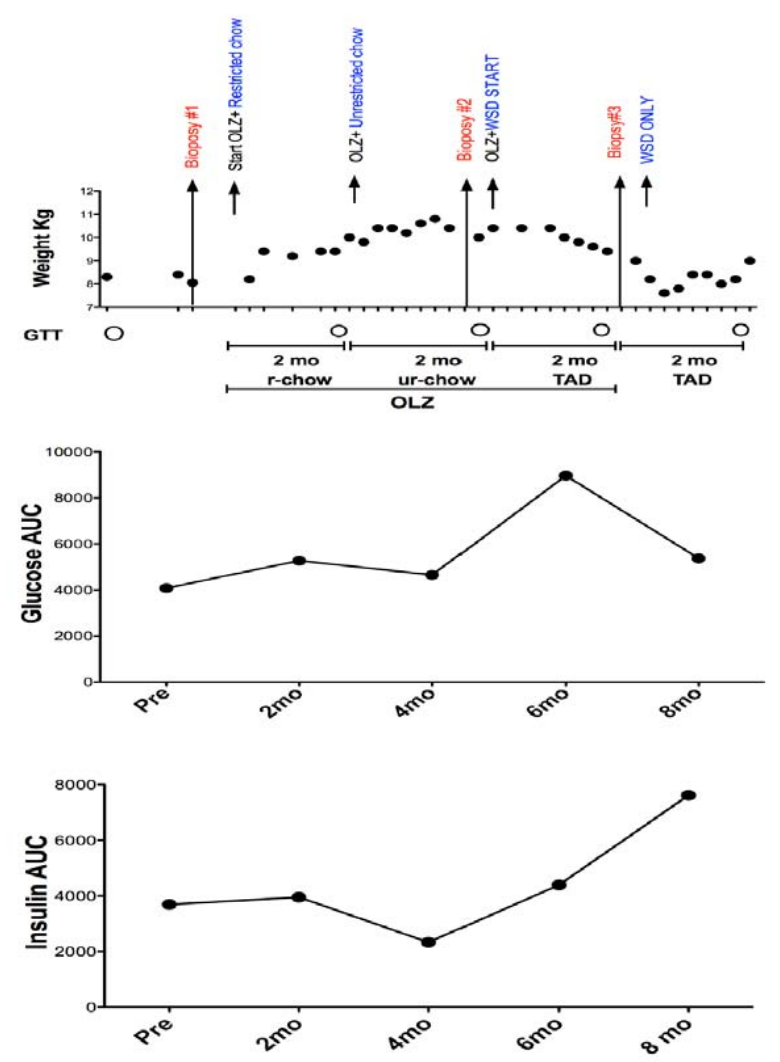

Figure 1: Illustration of the protocol for the preliminary data collection and the area under the curve for glucose and insulin in serial GTTs (circles). Biopsies are shown in red text and protocol changes are shown in blue text. 

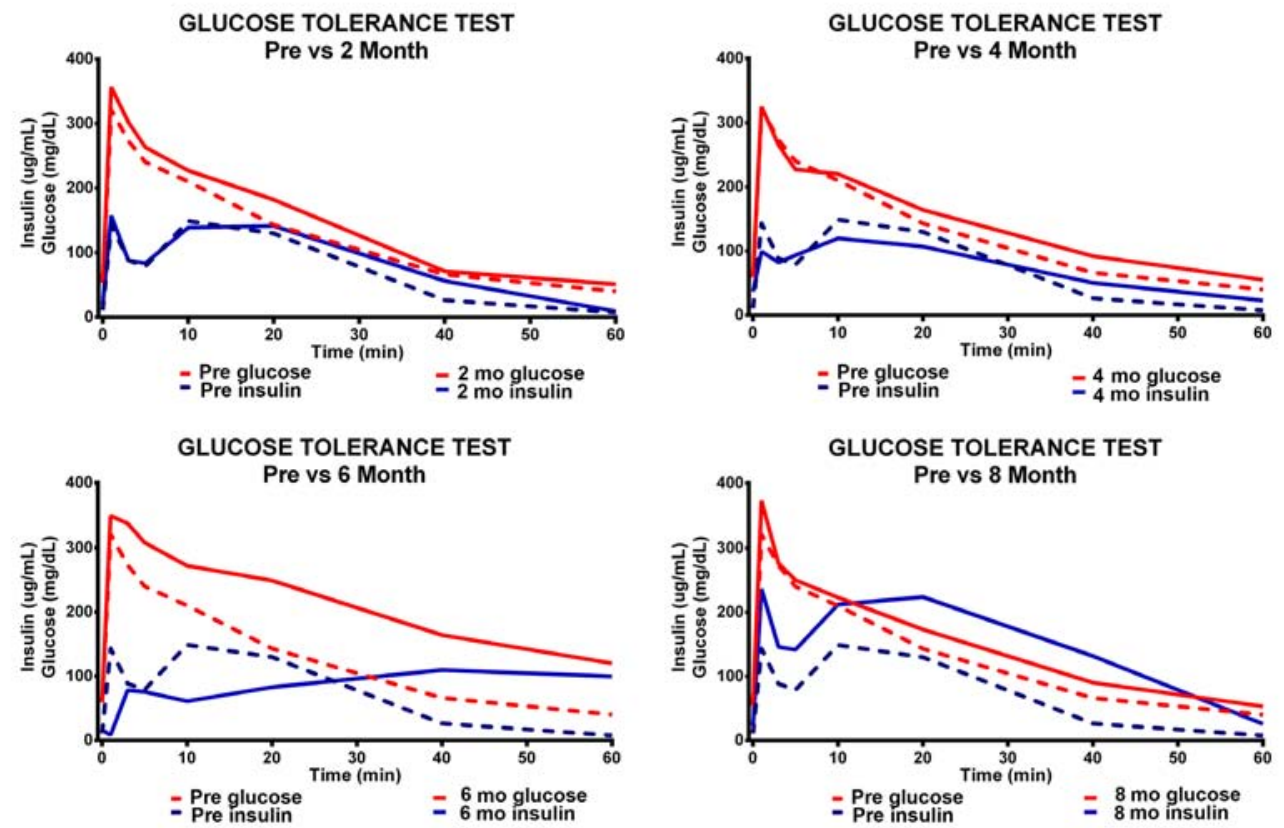

Figure 2: Serial GTTs. Dotted lines represent the baseline before treatment responses.

Table 1: Summary of Dexascan results in the female Japanese macaque treated with and without OLZ and WSD in a longitudinal manner.

\begin{tabular}{|l|c|c|c|c|c|c|}
\hline Treatment Group & BMC (g) & Fat $(\mathbf{g})$ & Lean $(\mathbf{g})$ & Lean+BMC (g) & Total Mass & \% Fat \\
\hline Baseline & 423 & 1157 & 6542 & 6965 & 8123 & 14.2 \\
\hline OLZ+Restricted chow & 433 & 2119 & 7139 & 7573 & 9693 & 21.9 \\
\hline OLZ+Unrestricted chow & 464 & 3116 & 6513 & 6977 & 10094 & 30.0 \\
\hline OLZ+WSD & 448 & 2588 & 6285 & 6733 & 9321 & 27.8 \\
\hline WSD & 424 & 1679 & 6751 & 7175 & 8855 & 19.0 \\
\hline
\end{tabular}

quantitative values for each curve are shown in Table 2. Glucose and insulin AUCs did not change with OLZ + Restricted chow (baseline vs. 2 mo) or OLZ + Unrestricted chow (baseline vs. 4 mo). Nonetheless, after unrestricted chow, first-phase insulin secretion was blunted. Addition of WSD caused an increase in glucose AUC (baseline vs. 6 mo), and no change in the insulin AUC, but there was a marked change in first-phase insulin secretion, and second-phase insulin secretion was elevated and prolonged. After OLZ withdrawal, but continuation of WSD, weight and glucose AUC declined, accompanied by markedly increased insulin AUC (pre vs. $8 \mathrm{mo}$ ). Although insulin levels were elevated, the pattern of first and second-phase insulin secretion appeared normal.

Examination of the GTT data (Figure 2) demonstrates in more detail that, at 2 mo after OLZ + Restricted chow, there was little change from baseline. However, with OLZ + Unrestricted chow (pre-vs $4 \mathrm{mo}$ ), there was an apparent decrease in firstphase insulin secretion. After 2 mo OLZ + WSD (pre vs. 6 mo), both first- and second-phase insulin secretion were reduced, and this was associated with increased post-prandial glucose. AUC glucose was elevated (pre vs. 6 mo GTT, Figure 2) indicating that clearance declined. After withdrawal of OLZ, but maintenance on
WSD (pre vs. 8 mo), the insulin secretory response was markedly elevated and this hyperinsulinemia restored glucose disposal. Fasting glucose did not change with the different treatments.

Dexascan (Table 1) showed that \% body fat increased from baseline with OLZ + Restricted chow and OLZ + Unrestricted chow. WSD did not change $\%$ body fat after 2 months. Removal of OLZ and continuation of the WSD reduced \% body fat. In addition, Dexascans showed that a predominant amount of fat was deposited in the midsection (Figure 3).

To evaluate adipose-specific effects, $\mathrm{OM}$ and SQ white adipose tissue biopsies were obtained at baseline (biopsy 1) and after OLZ + Unrestricted chow (biopsy 2) and after OLZ + WSD (biopsy 3) to evaluate adipocyte cell size, lipolysis, and insulin-stimulated FFA uptake (red type in Figure 1). As shown in Figure 4, adipocyte size was significantly increased by OLZ in both depots, with SC adipose tissue exhibiting a greater response, but WSD did not induce a further change.

Adipocyte hypertrophy (increased cell size) can affect lipolysis (represented by glycerol release into conditioned medium) under basal conditions or in response to the $\beta$-adrenergic agonist isoproterenol. At baseline, isoproterenol stimulated lipolysis in 
Table 2: Quantitative values (mg/dL) from the GTT curves at the two month time-point from the start of each treatment.

\begin{tabular}{|c|c|c|c|c|c|c|c|}
\hline Treatment Group & $\begin{array}{l}\text { Insulin } \\
\text { Start }\end{array}$ & $\begin{array}{c}\text { Glucose } \\
\text { Start }\end{array}$ & $\begin{array}{c}\text { Insulin } \\
\text { Peak Ph1 }\end{array}$ & $\begin{array}{c}\text { Insulin } \\
\text { Peak Ph2 }\end{array}$ & $\begin{array}{c}\text { Glucose } \\
\text { Peak }\end{array}$ & $\begin{array}{c}\text { Insulin } \\
\text { Area }\end{array}$ & $\begin{array}{c}\text { Glucose } \\
\text { Area }\end{array}$ \\
\hline Baseline chow & 10.9 & 59.0 & 142.9 & 148.7 & 321 & 3691 & 4075 \\
\hline OLZ+Unrestricted Chow & 36.7 & 62.0 & 99.2 & 119.6 & 325 & 2332 & 4649 \\
\hline OLZ+WSD & 15.6 & 60.0 & 77.9 & 109.4 & 350 & 4393 & $8964^{*}$ \\
\hline
\end{tabular}
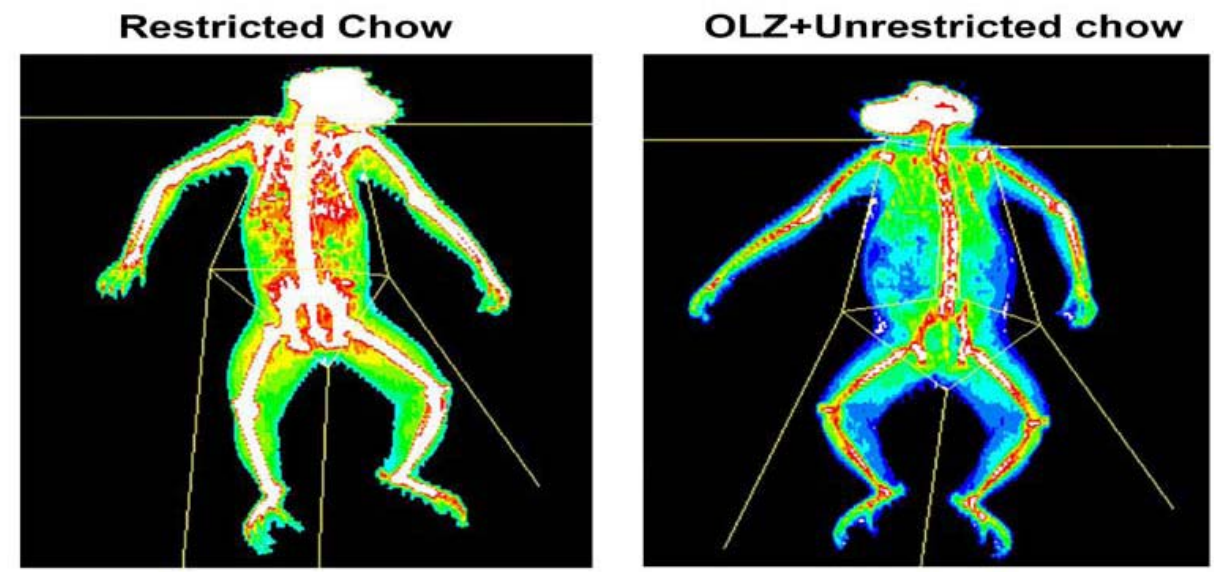

Figure 3: Dexascan pictures of the female Japanese macaque at baseline and after OLZ + Unrestricted chow. Note the increase in adiposity in the thoracic region.

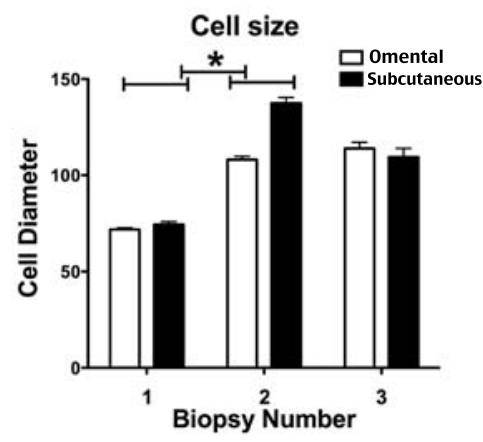

Figure 4: Adipose cell size at [1] baseline, and after [2] OLZ+chow and after [3] OLZ+WSD. There was a significant effect of fat location and biopsy number or in vivo treatment. There was also a significant interaction (all $p<0.0001,2$-way ANOVA). *Asterisks designate post hoc-pair wise differences (Newman-Keuls post hoc $p<0.05)$.

OM, but not in SQ fat. Exposure to OLZ increased isoproterenolstimulated lipolysis in both depots, but the in vivo addition of WSD with OLZ decreased isoproterenol-stimulated lipolysis in both depots (Figure 5).

As shown in Figure 6, insulin increased FFA uptake at baseline in both OM and SQ adipose tissue. OLZ + chow or OLZ + WSD increased basal FFA uptake in both OM and SQ adipose tissue (post hoc $p<0.05$ ), with insulin no longer inducing an additional significant increase, suggesting the early development of adipose insulin resistance.

A separate preliminary trial was conducted on two monkeys with 5 days of OLZ or no treatment followed by RT-PCR analysis of rostral preoptic (POA) and medial basal hypothalamus $(\mathrm{MBH})$ gene expression (Figure 7). OLZ treatment decreased pre-opiomelanocortin (POMC) mRNA in the MBH, indicating less alpha-melanocyte stimulating hormone ( $\alpha \mathrm{MSH})$ would be produced and less satiety achieved. OLZ increased expression of neuropeptide Y (NPY) and agouti-related peptide (AgRP) mRNAs, both coding for appetite stimulants. OLZ also increased expression for 5HT2C, MCR4 and leptin receptor (LepR) mRNAs.

\section{Discussion}

The in vivo data from this study suggest that OLZ immediately increased appetite and consumption of chow, but that induction of whole animal hyperglycemia required WSD as well. Withdrawal 
of OLZ allowed restoration of glucose clearance through increased insulin secretion. OLZ caused a rapid and marked change in hypothalamic gene expression related to satiety and feeding. POMC, the precursor to $\alpha \mathrm{MSH}$, which mediates satiety, was markedly down regulated. NPY and AgRP gene expression, which code for feeding peptides NPY and AgRP, were clearly increased. In the anterior hypothalamus where $\alpha$ MSH neurons project, there was an increase in gene expression for $5 \mathrm{HT} 2 \mathrm{C}$, MCR4, and LepR.

Adipocyte size was significantly increased with administration of OLZ for 4 months (mo), with a diet of normal monkey chow. Isoproterenol increased glycerol release when the animal was on monkey chow at baseline, or on OLZ + chow. However, with addition of WSD to OLZ, isoproterenol-induced glycerol release was significantly reduced, indicating a loss of sensitivity to adrenergic stimulation. Insulin stimulated FFA uptake at baseline in both OM and SQ fat. After 4 mo of OLZ + chow, insulin did not stimulate FFA uptake over the elevated basal uptake in either depot. The elevated basal FFA uptake may be attributable to the increase in cell size that occurred over 4 mo of OLZ treatment with

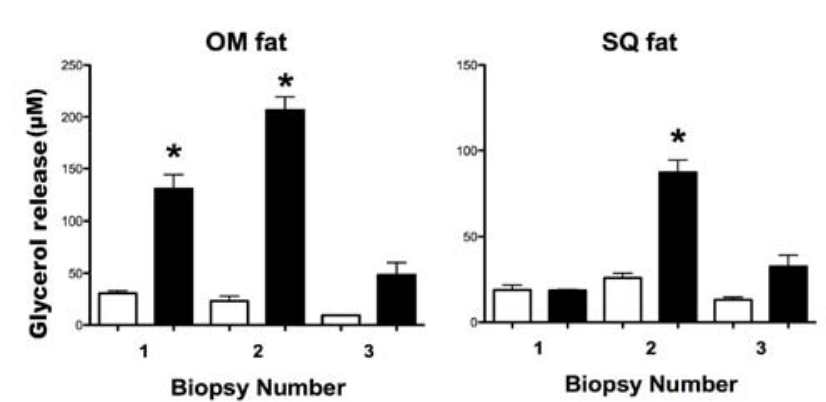

Figure 5: Isoproterenol-stimulated lipolysis at [1] baseline, [2] with OLZ+chow and with [3] OLZ+WSD. There was a significant difference between the groups with OM and SQ explants (both $p<0.0001$, ANOVA). *Asterisks designate post hoc pair-wise differences (Newman-Keuls post hoc $p<0.05$ ).
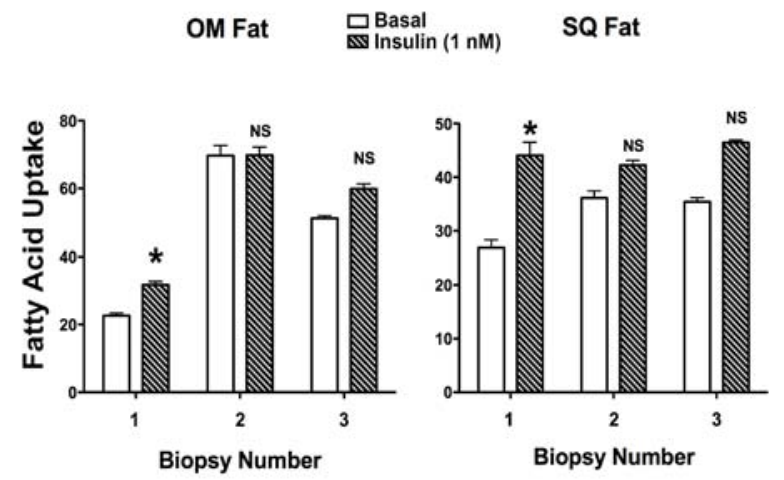

Figure 6: Isoproterenol-stimulated lipolysis at [1] baseline, [2] with OLZ+chow and with [3] OLZ+WSD. There was a significant difference between the groups with OM and SQ explants (both $p<0.0001$, ANOVA). *Asterisks designate post hoc pair-wise differences (Newman-Keuls post hoc $p<0.05$ ).

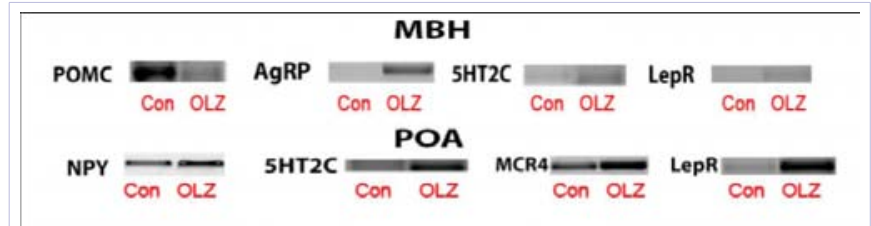

Figure 7: Semi-quantitative RT-PCR analysis of transcripts medial basal hypothalamus (MBH) and rostral hypothalamic preoptic area (POA). An untreated control (Con) monkey was compared to a monkey treated with OLZ for 5 days.

restricted or unrestricted chow. Thus, there was an indication of insulin resistance at the level of the adipocyte that preceded the hyperglycemia observed in the GTT after OLZ + WSD.

Clinical data show an increased diabetes risk in patients treated with clozapine or OLZ compared with untreated patients, and weight gain is more common in patients taking OLZ than with haloperidol or placebo [19]. Possible mechanisms include insulin resistance secondary to drug-induced weight gain, altered body fat distribution, or a direct effect on insulin-sensitive targets $[20,21]$. Our data support the involvement of all three. We have other data (not presented) that OLZ reduced insulinstimulated FFA uptake and this effect was reversed by addition of serotonin in 3T3-L1 adipocytes. Similar to our data obtained from experiments on the macaque monkey, OLZ reduced FFA uptake in rat peripheral tissues [22]. To date, studies in cell lines have shown negative effects of SGAs on adipocyte-type cell function, but this issue has not been adequately studied in humans with psychiatric illness [23].

An additional consequence of SGAs is their effect on serum lipids. Clozapine and OLZ, which produce the greatest weight gain, are associated with the greatest increase in levels of total cholesterol, LDL and triglycerides, and with decreased HDL cholesterol. Measurements of lipids, adiponectin or ghrelin were not obtained in this preliminary study, but there is reason to believe believe that the levels would be similar to that in humans humans. Future studies with more animals, and measurements of these and other endpoints, are awaiting funding.

Hypothalamic 5HT2C receptors play a major role in feeding and satiety [13]. Clinically, serotonergic agonists such as fenfluramine/phentermine (fen-phen) and, more recently, lorcaserin, a 5HT2C agonist (Belviq; Eisai/Arena Pharmaceuticals), have been used for weight reduction in morbid obesity. Two independent serotonin systems are now known to exist, one in the brain and the other in the periphery. Serotonin is a well-known central nervous system (CNS) neurotransmitter that regulates feeding behavior, meal size, and body weight [24]. Briefly, two populations of neurons in the arcuate nucleus (ARC) play primary roles in the regulation of eating. Neuropeptide $Y$ (NPY) and agouti-related peptide (AgRP) co-localizing neurons (NPY/AgRP) stimulate feeding, whereas POMC/CART colocalizing neurons mediate satiety and inhibit feeding [25-29]. Each of these populations is regulated by metabolic hormones such as insulin, leptin, and orexin, and by neurotransmitters such as serotonin [30]. POMC neurons produce the satiety peptide $\alpha \mathrm{MSH}$ via post-translational processing. In rodents, different 
populations of POMC neurons express 5HT2C and LepRs, which in turn, stimulate production and release of $\alpha$ MSH to decrease appetite as satiety is reached [24,31]. Our data indicate that by 5 days of OLZ treatment with a monkey chow diet, the expression of POMC is suppressed, whereas NPY and AgRP are markedly induced. Thus, OLZ has very rapid effects on the neural systems that govern feeding and satiety. There was also an increase in 5HT2C, MCR4 and LepR, possibly as an attempt to overcome OLZ antagonism and maintain homeostasis. Therefore, we hypothesize that the first action of OLZ is antagonism of 5HT2C receptors in hypothalamic neural systems, which causes an increase in appetite. The increase in the 5HT2C receptor expression indicates that antagonism of the $5 \mathrm{HT} 2 \mathrm{C}$ receptor by OLZ acts like serotonin denervation, and consequently results in the classical increase in expression of the postsynaptic receptors. The increase in MCR4 suggests a second classical homeostatic mechanism occurred to maintain satiety. Without serotonin stimulation of POMC neurons there would be decreased $\alpha \mathrm{MSH}$ neurotransmission that in turn leads to increased expression of MCR4. Interestingly, the increase in LepR occurred before weight gain and adiposity increased. This could be a third homeostatic mechanism in which the sensitivity to Leptin was increased to maintain POMC expression in the absence of serotonergic input. Nonetheless, these mechanisms were not sufficient to maintain normal body weight with continued administration of OLZ.

There is much less evidence for the role of serotonin as an endocrine hormone, particularly with respect to its effects on glucose and lipid metabolism. We now understand that murine pancreatic islets express serotonin system genes and that serotonin affects islet function [32-34]. Recent studies have shown that white adipose tissue also expresses serotonin receptors, tryptophan hydroxylase, and the serotonin reuptake transporter, and adipose cells secrete serotonin, which regulates leptin in mature adipocytes [35-37]. Furthermore, serotonin metabolites act as endogenous agonists for peroxisome proliferator-activated receptor (PPAR) $-\gamma$ and serotonin accelerates adipocyte differentiation via 5HT2A and $\mathrm{C}$ receptors $[36,38]$. Thus, serotonin acts in peripheral tissues central to insulin production and response.

Most studies of the peripheral effects of OLZ in the area of metabolism and serotonin have been conducted with rodents, but OLZ treatment has significantly different effects in rats and humans $[14,15]$. 5HT2A, 5HT2B, and 5HT2C receptors are expressed in adipose tissue $[35,36,39]$. Through these receptors, peripheral serotonin can modulate a variety of adipose functions, including adipocyte differentiation [36] and lipolysis [23]. By antagonizing serotonin action, OLZ and other SGAs impaired glucose and lipid disposal in fat and muscle [40], inhibited lipolysis, and increased lipogenesis in 3T3 adipocytes [23], consistent with our preliminary data. These properties of OLZ treatment correlate with the development of dyslipidemia and diabetes in the long term.

The results of the longitudinal GTTs indicated that induction of whole animal hyperglycemia required addition of WSD to OLZ; while withdrawal of OLZ allowed restoration of glucose clearance through increased insulin secretion. This observation raises the distinct possibility of a direct inhibitory action of OLZ on pancreatic $\beta$ cells. Beta cells have several serotonin receptors, and insulin co-localizes with serotonin [41]. Knock out of TPH1, with inhibition of peripheral serotonin production, causes $\beta$ cells to stop proliferating and leads to diabetes in adult mice [42]. It is attractive to speculate that serotonin is needed for optimum response of $\beta$ cells to glucose; and in the absence or antagonism of serotonin; the insulin response to glucose is severely blunted. The exact mechanism of serotonin action on the $\beta$ cell is unknown.

Our preliminary data suggest the development of local adipocyte insulin resistance and adipocyte hypertrophy followed 4 mo of OLZ treatment, which occurred on unrestricted chow at the time that whole animal GTT was normal. The possibility exists that the adipocyte insulin resistance was due to adipocyte hypertrophy, which was a consequence of weight gain, rather than a direct effect of OLZ. The ability of serotonin to reverse the effect of OLZ on insulin-induced FFA uptake in 3T3-L1 cells argues against this possibility as being entirely responsible. A recent study by Teff et al. [43] demonstrated that shortterm treatment of normal subjects with OLZ produced insulin resistance and hyperinsulinemia in conjunction with changes in glucagon and GLP-1 levels that were independent of weight gain [43]. This observation further supports the notion that OLZ has immediate direct effects on peripheral tissues. With comparison of the cellular actions and in vivo actions of OLZ, it appeared that insulin resistance manifested at the level of the adipocyte prior to manifestation in the GTTs. It is possible that muscle use of glucose delayed the onset of hyperglycemia in the whole animal, although it has been suggested that SGAs may block the glucose transporter in muscle [44].

In summary, these preliminary data in our NHP model support the hypotheses that [i]OLZ acts on NHP peripheral tissues as well as in the CNS; [ii] that rapid early changes in hypothalamic gene expression lead to decreased satiety and increased feeding that precede fat accumulation; [iii] that adipose tissue exhibits insulin resistance prior to alterations in glucose tolerance and insulin secretion; [iv] that addition of WSD to OLZ precipitates impaired glucose clearance without hyperinsulinemia; and [v] that removal of OLZ and continued WSD result in normalized glucose tolerance and increased insulin secretion. Our data suggest complex and early responses to OLZ that may be exacerbated by WSD, and which need confirmation with more animals. In addition, better treatments need to be developed that block the metabolic effects of OLZ while maintaining its psychiatric benefits.

\section{Acknowledgement}

Supported by NIH grants MH86542 to CLB and P51 OD011092 for the operation of ONPRC.

\section{References}

1. VanItallie TB. Worldwide epidemiology of obesity. Pharmacoeconomics. 1994; 5: 1-7.

2. Goff DC, Cather C, Evins AE, Henderson DC, Freudenreich O, Copeland PM, et al. Medical morbidity and mortality in schizophrenia: guidelines for psychiatrists. J Clin Psychiatry. 2005; 66(2): 183-194. 
3. Wu EQ, Birnbaum HG, Shi L, Ball DE, Kessler RC, Moulis M, et al. The economic burden of schizophrenia in the United States in 2002. J Clin Psychiatry. 2005; 66(9): 1122-9.

4. Casey DE. Extrapyramidal syndromes and new antipsychotic drugs: findings in patients and non-human primate models. Br J Psychiatry Suppl. 1996; (29): 32-9.

5. Casey DE. Pathophysiology of antipsychotic drug-induced movement disorders. J Clin Psychiatry. 2004; 65 Suppl 9: 25-8.

6. Davies MA, Compton-Toth BA, Hufeisen SJ, Meltzer HY, Roth BL. The highly efficacious actions of $\mathrm{N}$-desmethylclozapine at muscarinic receptors are unique and not a common property of either typical or atypical antipsychotic drugs: is M1 agonism a pre-requisite for mimicking clozapine's actions? Psychopharmacology (Berl). 2005; 178(4): 451-60.

7. Kroeze WK, Hufeisen SJ, Popadak BA, Renock SM, Steinberg S, Ernsberger P, et al. H1-histamine receptor affinity predicts shortterm weight gain for typical and atypical antipsychotic drugs. Neuropsychopharmacology. 2003; 28(3): 519-26.

8. Rauser L, Savage JE, Meltzer HY, Roth BL. Inverse agonist actions of typical and atypical antipsychotic drugs at the human 5-hydroxytryptamine(2C) receptor. J Pharmacol Exp Ther. 2001; 299(1): 83-9.

9. Han M, Deng C, Burne TH, Newell KA, Huang XF. Short- and longterm effects of antipsychotic drug treatment on weight gain and $\mathrm{H} 1$ receptor expression. Psychoneuroendocrinology. 2008; 33(5): 569580. doi: 10.1016/j.psyneuen.2008.01.018.

10. Simpson GM, Weiden P, Pigott T, Murray S, Siu CO, Romano SJ, et al Six-month, blinded, multicenter continuation study of ziprasidone versus olanzapine in schizophrenia. Am J Psychiatry. 2005; 162(8): 1535-8.

11. Nasrallah HA. Atypical antipsychotic-induced metabolic side effects: insights from receptor-binding profiles. Mol Psychiatry. 2008; 13(1): 27-35.

12. Newman-Tancredi A, Kleven MS. Comparative pharmacology of antipsychotics possessing combined dopamine D2 and serotonin 5-HT1A receptor properties. Psychopharmacology (Berl). 2011; 216(4): 451-473

13. Donovan MH, Tecott LH. Serotonin and the regulation of mammalian energy balance. Front Neurosci. 2013; 7: 36. doi: 10.3389/ fnins.2013.00036.

14. Cooper GD, Pickavance LC, Wilding JP, Harrold JA, Halford JC, Goudie AJ. Effects of olanzapine in male rats: enhanced adiposity in the absence of hyperphagia, weight gain or metabolic abnormalities. Psychopharmacol 2007; 21(4): 405-13.

15. Cooper GD, Harrold JA, Halford JC, Goudie AJ. Chronic clozapine treatment in female rats does not induce weight gain or metabolic abnormalities but enhances adiposity: implications for animal models of antipsychotic-induced weight gain. Prog Neuropsychopharmacol Biol Psychiatry. 2008; 32(2): 428-36.

16. Varlamov 0, Chu MP, McGee WK, Cameron JL, O’Rourke RW, Meyer KA et al. Ovarian cycle-specific regulation of adipose tissue lipid storage by testosterone in female nonhuman primates. Endocrinology. 2013; 154(11): 4126-35. doi: 10.1210/en.2013-1428.

17. Varlamov O, Somwar R, Cornea A, Kievit P, Grove KL, Roberts CT Jr. Single-cell analysis of insulin-regulated fatty acid uptake in adipocytes. Am J Physiol Endocrinol Metab. 2010; 299(3): E486-96. doi: 10.1152/ ajpendo.00330.2010
18. Varlamov O, White AE, Carroll JM, Bethea CL, Reddy A, Slayden O, et al. Androgen effects on adipose tissue architecture and function in nonhuman primates. Endocrinology. 2012; 153(7): 3100-10. doi: 10.1210/en.2011-2111.

19. Atmaca M, Kuloglu M, Tezcan E, Gecici O, Ustundag B. Weight gain, serum leptin and triglyceride levels in patients with schizophrenia on antipsychotic treatment with quetiapine, olanzapine and haloperidol. Schizophr Res 2003; 60(1): 99-100.

20. Irwin N, Gault VA. Unraveling the mechanisms underlying olanzapineinduced insulin resistance. Diabetes. 2013; 62(9): 3022-3. doi: $10.2337 / \mathrm{db} 13-0804$.

21. Newcomer JW. Second-generation (atypical) antipsychotics and metabolic effects: a comprehensive literature review. CNS Drugs. 2005; 19 Suppl 1: 1-93.

22. Albaugh VL, Vary TC, Ilkayeva O, Wenner BR, Maresca KP, Joyal JL, et al. Atypical antipsychotics rapidly and inappropriately switch peripheral fuel utilization to lipids, impairing metabolic flexibility in rodents. Schizophr Bull. 2012; 38(1): 153-66. doi: 10.1093/schbul/ sbq053.

23. Vestri HS, Maianu L, Moellering DR, Garvey WT. Atypical antipsychotic drugs directly impair insulin action in adipocytes: effects on glucose transport, lipogenesis, and antilipolysis. Neuropsychopharmacology. 2007; 32(4): 765-72

24. Lam DD, Przydzial MJ, Ridley SH, Yeo GS, Rochford JJ, O’Rahilly S et al. Serotonin 5-HT2C receptor agonist promotes hypophagia via downstream activation of melanocortin 4 receptors. Endocrinology. 2008; 149(3): 1323-8.

25. Kim MS, Rossi M, Abusnana S, Sunter D, Morgan DG, Small CJ, et al. Hypothalamic localization of the feeding effect of agouti-related peptide and alpha-melanocyte-stimulating hormone. Diabetes. 2000; 49(2): 177-82.

26. Kristensen P, Judge ME, Thim L, Ribel U, Christjansen KN, Wulff BS, et al. Hypothalamic CART is a new anorectic peptide regulated by leptin. Nature. 1998; 393(6680): 72-6

27. Higuchi $H$. Molecular analysis of central feeding regulation by neuropeptide Y (NPY) neurons with NPY receptor small interfering RNAs (siRNAs). Neurochem Int. 2012; 61(6): 936-41. doi: 10.1016/j. neuint.2012.02.029.

28. Luquet S, Perez FA, Hnasko TS, Palmiter RD. NPY/AgRP neurons are essential for feeding in adult mice but can be ablated in neonates. Science. 2005; 310(5748): 683-5.

29. Hadley ME, Haskell-Luevano C. The proopiomelanocortin system. Ann N Y Acad Sci. 1999; 885: 1-21.

30. Funahashi H, Yamada S, Kageyama H, Takenoya F, Guan JL, Shioda S, et al. Co-existence of leptin- and orexin-receptors in feeding-regulating neurons in the hypothalamic arcuate nucleus-a triple labeling study. Peptides. 2003; 24(5): 687-94.

31.Sohn JW, Xu Y, Jones JE, Wickman K, Williams KW, Elmquist Jk Serotonin 2C receptor activates a distinct population of arcuate proopiomelanocortin neurons via TRPC channels. Neuron. 2011; 71(3): 488-497. doi: 10.1016/j.neuron.2011.06.012.

32. Ohta Y, Kosaka Y, Kishimoto N, Wang J, Smith SB, Honig G, et al. Convergence of the insulin and serotonin programs in the pancreatic $\beta$-cell. Diabetes. 2011; 60(12): 3208-16. doi: 10.2337/db10-1192.

33. Kim H, Toyofuku Y, Lynn FC, Chak E, Uchida T, Mizukami H, et al. Serotonin regulates pancreatic beta cell mass during pregnancy. Nat Med. 2010; 16(7): 804-808. doi: 10.1038/nm.2173. 
34. Isaac R, Boura-Halfon S, Gurevitch D, Shainskaya A, Levkovitz Y, Zick Y. Selective serotonin reuptake inhibitors (SSRIs) inhibit insulin secretion and action in pancreatic $\beta$ cells. J Biol Chem. 2013; 288(8): 5682-93. doi: 10.1074/jbc.M112.408641.

35. Stunes AK, Reseland JE, Hauso O, Kidd M, Tommeras K, Waldum HL, et al. Adipocytes express a functional system for serotonin synthesis, reuptake and receptor activation. Diabetes Obes Metab. 2011; 13(6) 551-8. doi: 10.1074/jbc.M112.408641.

36. Kinoshita M, Ono K, Horie T, Nagao K, Nishi H, Kuwabara Y, et al Regulation of adipocyte differentiation by activation of serotonin (5HT) receptors 5-HT2AR and 5-HT2CR and involvement of microRNA448-mediated repression of KLF5. Mol Endocrinol. 2010; 24(10): 1978-87. doi: 10.1210/me.2010-0054.

37. Soliman MM. Modulation of cytokines, adiponectin and leptin expression in mature bovine adipocytes by serotonin. Australian J Basic Applied Sci 2010; 4: 1157-64.

38. Watanabe H, Rose MT, Aso H. Role of peripheral serotonin in glucose and lipid metabolism. Curr Opin Lipidol 2011; 22(3): 186-91. doi: 10.1097/MOL.0b013e3283462273.

39. Uchida-Kitajima S, Yamauchi T, Takashina Y, Okada-Iwabu M, Iwabu M, Ueki K, et al. 5-Hydroxytryptamine $2 \mathrm{~A}$ receptor signaling cascade modulates adiponectin and plasminogen activator inhibitor 1 expression in adipose tissue. FEBS Lett. 2008; 582(20): 3037- 44. doi: 10.1016/j.febslet.2008.07.044

40.Vidarsdottir S, de Leeuw van Weenen JE, Frölich M, Roelfsema F, Romijn JA, Pijl H. Effects of olanzapine and haloperidol on the metabolic status of healthy men. J Clin Endocrinol Metab. 2010; 95(1): 118-25. doi: 10.1210/jc.2008-1815.

41. Richmond JE, Codignola A, Cooke IM, Sher E. Calcium- and bariumdependent exocytosis from the rat insulinoma cell line RINm5F assayed using membrane capacitance measurements and serotonin release. Pflugers Arch. 1996; 432(2): 258-69.

42. Paulmann N, Grohmann M, Voigt JP, Bert B, Vowinckel J, Bader M, et al. Intracellular serotonin modulates insulin secretion from pancreatic beta-cells by protein serotonylation. PLoS Biol. 2009; 7(10): e1000229. doi: 10.1371/journal.pbio.1000229.

43. Teff KL, Rickels MR, Grudziak J, Fuller C, Nguyen HL, Rickels K. Antipsychotic-induced insulin resistance and postprandial hormonal dysregulation independent of weight gain or psychiatric disease. Diabetes. 2013; 62(9): 3232-40. doi: 10.2337/db13-0430.

44. Starrenburg FC, Bogers JP. How can antipsychotics cause Diabetes Mellitus? Insights based on receptor-binding profiles, humoral factors and transporter proteins. Eur Psychiatry. 2009; 24(3): 164-170. doi: 10.1016/j.eurpsy.2009.01.001. 